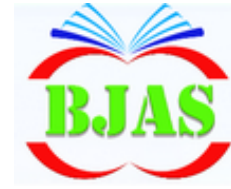

Available online at http://bajas.edu.iq https://doi.org/10.37077/25200860.2019.196 College of Agriculture, University of Basrah

ISSN 1814 - 5868

Basrah J. Agric. Sci., 32(2): 44-59, 2019

\section{Basrah Journal of \\ Agricultural Sciences}

\title{
Effect of Sodium Nitroprusside (SNP) on Minerals Content of Cabbage Brassica oleracea var. capitata L. Grown under Salt Stress
}

\author{
Talib M. M. Al-Jarah', Awatif N. Jerry ${ }^{2 *}$ \& Abbas M. Jasim² \\ 1 Agriculture Directorate of Maysan, Ministry of Agriculture, Iraq. \\ ${ }^{2}$ Department of Horticulture and Landscape Design -College of Agriculture \\ University of Basrah, Iraq. \\ *Corresponding author: a_n_jerry@yahoo.com
}

\section{Received 17 June 2019; Accepted 14 October 2019; Available online 30 December 2019}

\begin{abstract}
The experiment was conducted during the winter season of 2017-2018 and 20182019 at directorates of agriculture in Al-Zubair district, Basrah, Iraq to study the effect of sodium nitroprusside (SNP) at four concentration $(0,50,100$ and 150) $\mu \mathrm{M}$ with number of sprays (once and twice) and three cultivars (Pruktor F1, Luna and Rain ball F1) on mineral content of Nitrogen (N), Phosphorous (P), Potassium (K), Sodium (Na), Potassium/Sodium $\left(\mathrm{K}^{+} / \mathrm{Na}^{+}\right)$ratio, Chloride $\left(\mathrm{Cl}^{-}\right)$, Sulfur $(\mathrm{S})$ and Iron $(\mathrm{Fe})$ of leaves. Split-Split Plot design was used with three replicates per treatment. The means of treatments were compared by L.S.D. at 0.05 probability. Results indicated that Pruktor F1 had the highest percentage of N, P, K, $\mathrm{K}^{+} / \mathrm{Na}^{+}$ratio for both growing seasons while the highest accumulation of $\mathrm{Na}$ and $\mathrm{Cl}^{-}$in Rain ball F1 cultivar. Plants sprayed twice with SNP had a significant increase in $\mathrm{P}, \mathrm{K}, \mathrm{K}^{+} / \mathrm{Na}^{+}$ ratio, $\mathrm{S}$, and Fe comparing with once spray for both growing seasons. PruktorF1 sprayed with SNP at $100 \mu \mathrm{M}$ twice time was superior in $\mathrm{P}, \mathrm{K}, \mathrm{K}^{+} / \mathrm{Na}^{+}$ratio, $\mathrm{S}$ and $\mathrm{Fe}$, whereas, the same cultivar at $150 \mu \mathrm{M}$ concentration was superior in $\mathrm{N}$ for both growing seasons, same cultivar at 100 and $150 \mu \mathrm{M}$ sprayed twice time gave the lowest percentage of $\mathrm{Na}^{+}$and $\mathrm{Cl}^{-}$, respectively. Also Rain ball $\mathrm{F} 1$ at control treatment had the highest accumulation of $\mathrm{Na}$ and of $\mathrm{Cl}^{-}$.
\end{abstract}

Keywords: Sodium nitroprusside, Salt stress, Nitric oxide, Nitrogen, Phosphorus, Potassium.

\section{Introduction}

Cabbage belongs to the Cruciferae family and considers of the favourite winter crop in Iraq and other countries due to its using of salad and cooking, and it is planted in most regions of Iraq and the planted areas were reached 3315 Donums at 2018 with a total production of 6130 tons (Agricultural Statistics Directorate, 2018).

The salinity of irrigation water is one of the most important problems facing agriculture in arid and semi-arid region of the world and it is one of the main problems that faced the agricultural production in the desert region in Al-Zubair resulting in decreasing growth and photosynthesis efficiency, respiration and availability of nutrients and oxidation as a result of salt stress due to reactive oxygen species (ROS) (Munns \& Tester, 2008). Agriculture in Al-Zubair depends on the well waters because of the Lack of surface water, low rainfall and the random use of salty water 
leads to negative results on the availability of nutrients through the toxicity of $\mathrm{Na}^{+}$and $\mathrm{Cl}^{-}$ ions and the increasing of them with other ions leads to increase the osmotic pressure and imbalance the homeostasis nutrients, therefore methods must be applied in order to increase production and reduce environmental stress (Phocaides, 2001).

Cabbage is moderately sensitive to salinity and the salinity has caused negative effects on production (Ayers, \& Wescot, 1985), and it is necessary to use some techniques to reduce the influence of salt stress by using the SNP (donor NO), an inorganic compound $\left(\mathrm{Na}_{2}\left[\mathrm{Fe}(\mathrm{CN})_{5} \mathrm{NO}\right] .2 \mathrm{H}_{2} \mathrm{O}\right)$ which used to cure from heart disease for human by expanding vascular and it is essential drugs (WHO, 2015). It is one of the important technique which used for regulating most of physiological processes, including the responses to biotic and abiotic stress and increase the tolerance to salt stress by stimulating the antioxidant enzymes, which play a vital role in protection system and increase their tolerance to salt stress resulting in homeostasis nutrients and ionic balance (Nabi et al., 2019; Santisree et al., 2019).

Many studies referred to the spray with SNP compound for plants under salt stress (Molassiotis et al., 2010) and Nitric oxide (NO) increase the activity of plasma membrane $\mathrm{H}^{+}$-ATPase in plant exposed to salt stress and led to increase in $\mathrm{K}^{+} / \mathrm{Na}^{+}$ratio in the tissue of plants to increase adaptation to salt stress (Zhao et al., 2004). NO has a capability to remove reactive oxygen species(ROS) and acts as antioxidant by altering the gene expression of antioxidants and thus protect cells from oxidative damage caused by salt stress (Arasimowicz \& Floryszak-Wieczorek, 2007).
An experiment aimed to study the effect of SNP and the number of sprays on leaf $\mathrm{N}, \mathrm{P}$, $\mathrm{K}, \mathrm{Na}, \mathrm{K}+\mathrm{Na}+$ ratio, $\mathrm{Cl}-, \mathrm{S}$ and $\mathrm{Fe}$ content for three cultivars of cabbage under salt stress in the desert region southern Iraq.

\section{Materials \& Methods}

\section{A-field experiment}

The experiment was carried out during the winter season of 2017-2018 and 2918-2019 in the tomato development project at Al-Zubair, Directorate of Agriculture of Basrah. Random samples of field soil was taken to estimate some of the chemical and physical properties of it (table 1) and table (2) showed some chemical and physical properties of irrigation water (well water). The soil was plowed twice Perpendicular to a depth $30 \mathrm{~cm}$ and left for a month, the field arranged in 18 lines 28.8 meters long and $40 \mathrm{~cm}$ wide and $40 \mathrm{~cm}$ between the lines and every line was divided into four experimental units with a length 7.2 meter. The field soil fertilized with an organic fertilizer at the rate of 13 tone.Donum ${ }^{-1}$ and decomposed fertilizer NPK (15: 15: $15+$ TE) at the rate $0.5 \mathrm{~kg}$ for every unit and covered with the soil of the field with at $10 \mathrm{~cm}$ layer. The field was supplied with a drip irrigation system and the line covered with black plastic mulching.

The seeds were sown in styropor trays with 209 holes and were sterilized with beltanol $50 \%$ SL and filled with German peat moss, at 10/9/2017 and 9/9/2018 for both growing seasons. The seedlings were transplanted to the field after 45 days from sowing. First spray with the SNP after two weeks of transplanting and second spray after two 
Table (1): Physical and chemical properties of the field soil.

\begin{tabular}{|c|c|c|c|c|}
\hline \multicolumn{2}{|c|}{ Properties of the soil } & 2017-2018 Season & $\begin{array}{c}2018-2019 \\
\text { Season }\end{array}$ & Type of analysis \\
\hline \multirow{2}{*}{\multicolumn{2}{|c|}{$\frac{\text { Electrical conductivity }\left(\mathrm{ds} \cdot \mathrm{m}^{-1}\right)}{\text { Soil } \mathrm{pH}}$}} & 7.11 & 7.40 & \multirow{2}{*}{$\begin{array}{l}\text { Page } \text { et al. } \\
\text { (1982) }\end{array}$} \\
\hline & & 7.31 & 7.55 & \\
\hline \multirow{6}{*}{$\begin{array}{l}\text { Soluble ions } \\
\quad(\mathrm{mM})\end{array}$} & $\mathrm{Na}^{+}$ & 24.0 & 30.0 & \\
\hline & $\mathrm{Ca}^{++}$ & 17.75 & 20.00 & \multirow{2}{*}{ Richards (1954) } \\
\hline & $\mathrm{Mg}^{++}$ & 15.0 & 10.5 & \\
\hline & $\mathrm{SO}_{4}^{-}$ & 19.62 & 23.14 & $\begin{array}{c}\text { Page et al. (1982 } \\
\text { ) }\end{array}$ \\
\hline & $\mathrm{Cl}^{-}$ & 67.00 & 65.00 & Jackson (1958) \\
\hline & $\mathrm{HCO}_{3}^{-}$ & 2.6 & 2.8 & Richards (1954) \\
\hline Available Nitrogen & \multirow{3}{*}{$\mathrm{mg} \cdot \mathrm{Kg}^{-1}$} & 154 & 170 & \multirow{4}{*}{$\begin{array}{c}\text { Page et al. (1982 } \\
\text { ) }\end{array}$} \\
\hline Available phosphorus & & 69.02 & 75.40 & \\
\hline Available potassium & & 201.0 & 185.4 & \\
\hline Organic matter & $\left.\mathrm{Kg}^{-1}\right)$ & 4.64 & 5.08 & \\
\hline \multicolumn{5}{|c|}{ Soil structure } \\
\hline Sand & & 83.0 & 83.0 & \multirow{4}{*}{ Black (1965) } \\
\hline Silt & & 3.6 & 3.6 & \\
\hline Clay & & 13.4 & 13.4 & \\
\hline Soil textur & & $\begin{array}{l}\text { Loamy sand } \\
\text { texture }\end{array}$ & & \\
\hline
\end{tabular}

Table (2): Physical and chemical properties of irrigation water (well water).

\begin{tabular}{|c|c|c|c|}
\hline Properties of the water & Unit & 2017-2018 Season & 2018-2019 Season \\
\hline $\mathrm{pH}$ & - & 7.11 & 7.35 \\
\hline Electrical conductivity ( EC ) & ds. $\mathrm{m}^{-1}$ & 11.68 & 16.55 \\
\hline $\mathrm{Ca}^{++}$ & $\mathrm{mg} \cdot \mathrm{L}^{-1}$ & 570.0 & 480.0 \\
\hline $\mathrm{Mg}^{++}$ & & 409.9 & - \\
\hline $\mathrm{Na}^{+}$ & & 870.9 & 1009.0 \\
\hline $\mathrm{K}^{+}$ & & 40.5 & 55.2 \\
\hline $\mathrm{Cl}^{-}$ & & 177.2 & 211.0 \\
\hline $\mathrm{NO}_{3}^{-}$ & & 8.0 & 9.0 \\
\hline $\mathrm{SO}_{4}^{-}$ & & 795.0 & 850.0 \\
\hline
\end{tabular}

weeks from the first spray for Pruktor F1, Luna and Rain ball F1 with concentration $0,50,100$ and $150 \mu \mathrm{M}$.

The treatments were assigned to a completely randomized block design in a split- split plot arrangement with the cultivar in the main plots and spray time in the sub- plots andfoliar sprays of SNP in sub-sub plots with three replicates.
The means of treatments were compared by L.S.D. at 0.05 probability.

B-Determination of leaves content of nutrient elements:

1-Total nitrogen ( $\mathrm{N} \%$ ) was determined by micro-kjeldalmethod according to Page et al. (1982).

2-Phosphorus (P \%) was determined according to the method described by Jakson (1985). 
3-Potassium and Sodium (K \%, $\mathrm{Na} \%$ ) were determined according to the method described by Page et al. (1982).

$4-\mathrm{K}^{+} / \mathrm{Na}^{+}$ratio by dividing $\mathrm{K}^{+} \%$ on $\mathrm{Na}^{+}$ $\%$.

5-Chloride $\left(\mathrm{Cl}^{-} \%\right)$ was determined according to the method described by Furman (1962).

6-Sulfur (S\%) was determined according to a method described by Novozamsky \& Eck (1977).

7-Iron (Fe mg.kg-1) was determined by using atomic absorption according to a method described by Haynes (1980).

\section{Results \& Discussion}

Data presented in tables (3-9) explained that Pruktor F1 cultivar had a significant increase in $\mathrm{N}, \mathrm{P}, \mathrm{K}, \mathrm{K}^{+} / \mathrm{Na}^{+}$ratio, $\mathrm{S}$ and $\mathrm{Fe}$ and a significant decrease in $\mathrm{Na}^{+}$and $\mathrm{Cl}^{-}$ compared to other cultivars. Plants sprayed with SNP had a significant increase in the contents_of $\mathrm{N}, \mathrm{P}, \mathrm{K}, \mathrm{K}^{+} / \mathrm{Na}^{+}$ ratio, $\mathrm{S}$ and $\mathrm{Fe}$ compared with control treatment and significant decrease in $\mathrm{Na}^{+}$ and $\mathrm{Cl}^{-}$. Plants which sprayed twice with SNP at $100 \mu \mathrm{M}$ had the best result in $\mathrm{P}, \mathrm{K}$, $\mathrm{K}^{+} / \mathrm{Na}^{+}$ratio and $\mathrm{Fe}$. Plants which sprayed once with SNP at $150 \mu \mathrm{M}$ had significant increases in $\mathrm{N}$ comparing with control treatment.

The Pruktor F1 cultivar plants that sprayed twice with SNP at $100 \mu \mathrm{M}$ had highest values of $\mathrm{P}, \mathrm{K}, \mathrm{K}^{+} / \mathrm{Na}^{+}$ratio $\mathrm{S}$ and $\mathrm{Fe}$ and lowest values of $\mathrm{Cl}^{-}$. the same cultivar which sprayed once with $150 \mu \mathrm{M}$ SNP had gave highest $\mathrm{N}$ and lowest values of $\mathrm{Na}$, while untreated Rain ball $\mathrm{F}$ 1 cultivar gave less $\mathrm{N}, \mathrm{P}, \mathrm{K}, \mathrm{K}^{+} / \mathrm{Na}^{+}$ratio, $\mathrm{S}$ and $\mathrm{Fe}$ and highest values of $\mathrm{Na}$ and $\mathrm{Cl}^{-}$ for both growing seasons, respectively.
The results presented in table Tables (3, 4, 5, 7, 9 \& 10) revealed that Pruktor F1 cultivar was superior compared to other cultivars. Genetic factors of cultivars and its ability to prevent sodium ion uptake to plant roots. Results exhibited accumulation of nutrient elements except for $\mathrm{Na}^{+}$and $\mathrm{Cl}^{-}$ when the SNP used well water with high salt $(11.68,16.55) \mathrm{ds} . \mathrm{m}^{-1}$ for both growing seasons (table, 2). Spraying SNP twice had a significant effect (tables 4-5 \& 7) than once spray (tables 3, $6 \& 8$ ) because of the high concentration had unaffected role, so it exhibited the growth (Hayat et al., 2014) and due to joint interaction with ROS, which causes damage and breakdown of cells in more than one location, including the cellular membrane which causes oxidative stress (Belgini \& Lamattina, 1999).

Tables (3-5, 7 \& 9$)$ revealed a significant decrease in the content of $\mathrm{N}, \mathrm{P}$, $\mathrm{K}, \mathrm{K}^{+} / \mathrm{Na}^{+}$ratio, $\mathrm{S}$ and $\mathrm{Fe}$ when irrigated with salty water compared with the an increase of $\mathrm{N}^{+}$and $\mathrm{Cl}^{-}$ions. This lack of nitrogen content (table 3 ) caused a decrease in protein because of decreasing the activity of Nitrate reductase enzyme which affected of the synthesis of protein and total nitrogen (Lopez-Cantarero et al., 1997; Jabeen \& Ahmad, 2011), and the lack of water stimulates protease enzyme (Reddy \& Vora, 1985) and the competition between chloride and nitrate ion the exhibited nitrate and transporter because of toxic effect of salt ions (Lin et al., 1997) that led to accumulation of $\mathrm{Cl}^{-}$in leaves (Dean-Drummond, 1986) and it may also be due to the change in permeability properties of the plasma membrane, which 
affected by membrane proteins (Meloni et al., 2004).

The decrease of $\mathrm{P}$ in untreated plants (Table 4) were due to the competition between $\mathrm{Cl}^{-}$and $\mathrm{H}_{2} \mathrm{PO}_{4}^{-}$ions that $\mathrm{Cl}^{-}$ion acts on decrease absorption $\mathrm{H}_{2} \mathrm{PO}_{4}^{-}$by the plant (Pessarkli, 1999) and the reducing of phosphorous translocation from root to vegetative growth (Martinez \& Luchli, 1994). The salinity leads to reduction of root growth and its movement in soil and because of limiting movement of $\mathrm{P}$ lead $\mathrm{s}$ to reduce its adsorption (Al-Taey et al., 2017). The reduction of $K$ (Table 5) in untreated plants were due to the competition between $\mathrm{Na}^{+}$and $\mathrm{K}^{+}$ion on the absorption sites in roots and transporter proteins which transport $\mathrm{Na}^{+}$lasted of $\mathrm{K}^{+}$ (Ashley et al., 2006).

Data presented in table (6) revealed the reduction in $\mathrm{K}^{+}$in untreated plants because of well water that lead to reduce $\mathrm{K}^{+}$ absorption because of its effect on cellular organelles and transporter of $\mathrm{Na}^{+}$and $\mathrm{K}^{+}$ and $\mathrm{H}^{+}$pumps which generate the transporter forces ion in the cells (Zhu, 2003) and the reduction in water absorption was due to the high osmotic pressure in zone root that lead to reduced absorption on $\mathrm{K}^{+}$ion (Cuartero \& Fernande-Munoz, 1999) and that leads to reduction in the $\mathrm{K}^{+} / \mathrm{Na}^{+}$ratio (Table 7).

The high percentage of $\mathrm{Na}^{+}$and $\mathrm{Cl}^{-}$in untreated control (Table $6 \& 8$ ) because of their high concentration in a growth medium that lead to an increase of their absorption in plants.

Data presented in table (9) explained a significant decrease in $\mathrm{S}$ in untreated plants were due to the high salt in soil that lead to alkaline $\mathrm{pH}$ and saturated with calcium ion (Dougrameji \& Al-Rawi, 1972) and that effect on the availability of nutrients which important for plant growth. There was a significant decrease in the Iron element of untreated plants (Table, 10) because the irrigation water salinity lead to change the $\mathrm{pH}$ in soil and $\mathrm{Fe}$ sensitive to $\mathrm{pH}$ and that lead to reduce its availability for plant and then reduce its level in leaves and there was another reason of the toxic effect of $\mathrm{Na}^{+}$and $\mathrm{Cl}^{-}$on plasma membrane of root cells, which lead to reduce its ability for absorption and finally on absorption of nutrient elements like Fe (Passarakli, 1999).

Tables (3-5 \& 7) showed a significant increase in the percentage of nutrient elements which treated with the SNP because NO plays a vital role in alleviating of high salt in plant tissue and reduce the nutrient absorption. SNP leads to increase gene expression to $\mathrm{H}^{+}$-ATPase in plasma membrane and that lead to raising $\mathrm{K}^{+} / \mathrm{Na}^{+}$ in cells cytoplasm (Zhao et al., 2004) and showed the addition of SNP on Zea mays caused increasing the activity of $\mathrm{H}^{+}$ATPase in tonoplast and $\mathrm{Na}^{+} / \mathrm{H}^{+}$ transporter and enable $\mathrm{Na}^{+}$for passing and SNP improve macronutrient elements contents like Fe (Grazianoet al., 2002).

NO plays a physiological role in improving the transport of Fe from root to vegetative system, also, it made many nutrient elements can be absorbed by Ironregulated transporter 1(IRT1), which lead to an increase gene expression for them by NO (Connolly et al., 2002). 
Al-Jarah et Basrah al. / J. Agric. Sci., 32 (2): 47-62, 2019

Table (3): Effect of cultivars, sprays and concentration of SNP on N-content (\%) in leaves.

\begin{tabular}{|c|c|c|c|c|c|c|c|c|c|}
\hline \multirow{3}{*}{$\begin{array}{l}\text { SNP } \\
\mu \mathrm{M}\end{array}$} & \multirow[b]{3}{*}{ Sprays } & \multicolumn{4}{|c|}{$2017-2018$ Season } & \multicolumn{4}{|c|}{$2018-2019$ Season } \\
\hline & & \multicolumn{3}{|c|}{ Cultivars } & \multirow[b]{2}{*}{$\begin{array}{l}\text { SNP } \times \\
\text { Sprays }\end{array}$} & \multicolumn{3}{|c|}{ Cultivars } & \multirow[b]{2}{*}{$\begin{array}{l}\text { SNP } \times \\
\text { Sprays }\end{array}$} \\
\hline & & $\begin{array}{l}\text { Prukt } \\
\text { or F1 }\end{array}$ & Luna & $\begin{array}{c}\text { Rain } \\
\text { ball } \\
\text { F1 }\end{array}$ & & $\begin{array}{l}\text { Prukt } \\
\text { or F1 }\end{array}$ & Luna & $\begin{array}{c}\text { Rain } \\
\text { ball } \\
\text { F1 }\end{array}$ & \\
\hline \multirow{2}{*}{0} & $\begin{array}{l}\text { Once } \\
\text { time }\end{array}$ & 2.96 & 2.77 & 2.44 & 2.73 & 2.91 & 2.75 & 2.38 & 2.61 \\
\hline & $\begin{array}{l}\text { Twice } \\
\text { time }\end{array}$ & 2.99 & 2.81 & 2.49 & 2.76 & 2.97 & 2.79 & 2.43 & 2.64 \\
\hline \multirow{2}{*}{50} & $\begin{array}{l}\text { Once } \\
\text { time }\end{array}$ & 3.27 & 3.14 & 2.99 & 3.13 & 3.45 & 3.11 & 2.91 & 3.03 \\
\hline & $\begin{array}{l}\text { Twice } \\
\text { time }\end{array}$ & 3.84 & 3.38 & 3.35 & 3.52 & 3.43 & 3.34 & 3.27 & 3.44 \\
\hline \multirow{2}{*}{100} & $\begin{array}{l}\text { Once } \\
\text { time }\end{array}$ & 3.64 & 3.35 & 3.24 & 3.41 & 3.77 & 3.30 & 3.25 & 3.35 \\
\hline & $\begin{array}{l}\text { Twice } \\
\text { time }\end{array}$ & 3.51 & 3.19 & 3.14 & 3.28 & 3.51 & 3.16 & 3.11 & 3.23 \\
\hline \multirow{2}{*}{150} & $\begin{array}{l}\text { Once } \\
\text { time }\end{array}$ & 4.08 & 3.69 & 3.59 & 3.78 & 4.03 & 3.65 & 3.49 & 3.67 \\
\hline & $\begin{array}{l}\text { Twice } \\
\text { time }\end{array}$ & 3.46 & 3.15 & 3.14 & 3.25 & 3.45 & 3.16 & 3.08 & 3.18 \\
\hline \multicolumn{2}{|c|}{ LSD 0.05} & & 0.09 & & 0.05 & & 0.09 & & 0.06 \\
\hline \multicolumn{2}{|c|}{ Effect of cultivars } & 3.47 & 3.19 & 3.05 & \multirow{2}{*}{$\begin{array}{l}\text { Effect } \\
\text { of SNP }\end{array}$} & 3.34 & 3.10 & 2.99 & \multirow{2}{*}{$\begin{array}{l}\text { Effect } \\
\text { of SNP }\end{array}$} \\
\hline LSD & 0.05 & & 0.02 & & & & 0.03 & & \\
\hline \multirow{4}{*}{$\begin{array}{c}\text { Cultiva } \\
\text { rs } \times \\
\text { SNP }\end{array}$} & 0 & 2.98 & 2.79 & 2.46 & 2.74 & 2.81 & 2.67 & 2.38 & 2.62 \\
\hline & 50 & 3.55 & 3.26 & 3.17 & 3.33 & 3.44 & 3.17 & 3.09 & 3.23 \\
\hline & 100 & 3.57 & 3.27 & 3.19 & 3.35 & 3.47 & 3.22 & 3.18 & 3.29 \\
\hline & 150 & 3.77 & 3.42 & 3.37 & 3.52 & 3.64 & 3.34 & 3.30 & 3.43 \\
\hline \multicolumn{2}{|c|}{ LSD 0.05} & & 0.06 & & 0.04 & & 0.05 & & 0.03 \\
\hline & & & & & $\begin{array}{c}\text { Effect } \\
\text { of } \\
\text { Sprays }\end{array}$ & & & & $\begin{array}{c}\text { Effect } \\
\text { of } \\
\text { Sprays } \\
\end{array}$ \\
\hline \multirow{2}{*}{$\begin{array}{l}\text { Cultiva } \\
\text { rs } \times \\
\text { Sprays }\end{array}$} & $\begin{array}{l}\text { Once } \\
\text { time }\end{array}$ & 3.47 & 3.24 & 3.06 & 3.26 & 3.36 & 3.14 & 3.00 & 3.17 \\
\hline & $\begin{array}{l}\text { Twice } \\
\text { time }\end{array}$ & 3.45 & 3.13 & 3.03 & 3.21 & 3.33 & 3.06 & 2.97 & 3.12 \\
\hline \multicolumn{2}{|c|}{ LSD 0.05} & & 0.03 & & 0.02 & & $* \mathrm{NS}$ & & NS \\
\hline
\end{tabular}

*NS: not significant 
Al-Jarah et Basrah al. / J. Agric. Sci., 32 (2): 47-62, 2019

Table (4): Effect of cultivars, sprays and concentration of SNP on P-content (\%) in leaves.

\begin{tabular}{|c|c|c|c|c|c|c|c|c|c|}
\hline \multirow{3}{*}{$\begin{array}{l}\text { SNP } \\
\mu \mathrm{M}\end{array}$} & \multirow[b]{3}{*}{ Sprays } & \multicolumn{4}{|c|}{$2017-2018$ Season } & \multicolumn{4}{|c|}{$2018-2019$ Season } \\
\hline & & \multicolumn{3}{|c|}{ Cultivars } & \multirow[b]{2}{*}{$\begin{array}{l}\text { SNP } \times \\
\text { Sprays }\end{array}$} & \multicolumn{3}{|c|}{ Cultivars } & \multirow[b]{2}{*}{$\begin{array}{l}\text { SNP } \times \\
\text { Sprays }\end{array}$} \\
\hline & & $\begin{array}{l}\text { Prukt } \\
\text { or F1 }\end{array}$ & Luna & $\begin{array}{c}\text { Rain } \\
\text { ball } \\
\text { F1 }\end{array}$ & & $\begin{array}{c}\text { Prukto } \\
\text { r F1 }\end{array}$ & Luna & $\begin{array}{c}\text { Rain } \\
\text { ball } \\
\text { F1 }\end{array}$ & \\
\hline \multirow{2}{*}{0} & $\begin{array}{l}\text { Once } \\
\text { time }\end{array}$ & 0.330 & 0.302 & 0.280 & 0.304 & 0.314 & 0.284 & 0.269 & 0.289 \\
\hline & $\begin{array}{l}\text { Twice } \\
\text { time }\end{array}$ & 0.329 & 0.306 & 0.284 & 0.306 & 0.310 & 0.293 & 0.275 & 0.293 \\
\hline \multirow{2}{*}{50} & $\begin{array}{l}\text { Once } \\
\text { time }\end{array}$ & 0.383 & 0.356 & 0.333 & 0.358 & 0.364 & 0.341 & 0.322 & 0.342 \\
\hline & $\begin{array}{l}\text { Twice } \\
\text { time }\end{array}$ & 0.466 & 0.434 & 0.411 & 0.437 & 0.452 & 0.417 & 0.400 & 0.423 \\
\hline \multirow{2}{*}{100} & $\begin{array}{l}\text { Once } \\
\text { time }\end{array}$ & 0.441 & 0.413 & 0.393 & 0.415 & 0.423 & 0.398 & 0.380 & 0.401 \\
\hline & $\begin{array}{l}\text { Twice } \\
\text { time }\end{array}$ & 0.564 & 0.525 & 0.496 & 0.528 & 0.540 & 0.507 & 0.487 & 0.512 \\
\hline \multirow{2}{*}{150} & $\begin{array}{l}\text { Once } \\
\text { time }\end{array}$ & 0.504 & 0.456 & 0.451 & 0.470 & 0.481 & 0.438 & 0.438 & 0.452 \\
\hline & $\begin{array}{l}\text { Twice } \\
\text { time }\end{array}$ & 0.459 & 0.479 & 0.440 & 0.459 & 0.444 & 0.462 & 0.439 & 0.448 \\
\hline \multicolumn{2}{|c|}{ LSD 0.05} & & 0.012 & & 0.007 & & 0.015 & & 0.008 \\
\hline \multicolumn{2}{|c|}{ Effect of cultivars } & 0.434 & 0.409 & 0.386 & Effect & 0.416 & 0.392 & 0.376 & Effect \\
\hline \multicolumn{2}{|c|}{ LSD 0.05} & & 0.006 & & of SNP & & 0.009 & & of SNP \\
\hline \multirow{4}{*}{$\begin{array}{c}\text { Cultiva } \\
\text { rs } \times \\
\text { SNP }\end{array}$} & 0 & 0.329 & 0.304 & 0.282 & 0.305 & 0.312 & 0.288 & 0.272 & 0.291 \\
\hline & 50 & 0.425 & 0.395 & 0.372 & 0.397 & 0.408 & 0.379 & 0.361 & 0.383 \\
\hline & 100 & 0.503 & 0.469 & 0.444 & 0.472 & 0.482 & 0.453 & 0.434 & 0.456 \\
\hline & 150 & 0.481 & 0.468 & 0.445 & 0.465 & 0.463 & 0.450 & 0.438 & 0.450 \\
\hline \multicolumn{2}{|c|}{ LSD 0.05} & & 0.009 & & 0.005 & & 0.011 & & 0.006 \\
\hline & & & & & $\begin{array}{l}\text { Effect } \\
\text { of } \\
\text { Sprays }\end{array}$ & & & & $\begin{array}{c}\text { Effect } \\
\text { of } \\
\text { Sprays }\end{array}$ \\
\hline \multirow{2}{*}{$\begin{array}{c}\text { Cultiva } \\
\text { rs } \times \\
\text { Sprays }\end{array}$} & $\begin{array}{l}\text { Once } \\
\text { time }\end{array}$ & 0.414 & 0.382 & 0.364 & 0.387 & 0.395 & 0.365 & 0.352 & 0.371 \\
\hline & $\begin{array}{l}\text { Twice } \\
\text { time }\end{array}$ & 0.455 & 0.436 & 0.408 & 0.433 & 0.437 & 0.420 & 0.400 & 0.419 \\
\hline \multicolumn{2}{|c|}{ LSD 0.05} & & 0.006 & & 0.003 & & NS & & 0.004 \\
\hline
\end{tabular}


Table (5): Effect of cultivars, sprays and concentration of SNP on K content (\%) in leaves.

\begin{tabular}{|c|c|c|c|c|c|c|c|c|c|}
\hline \multirow{3}{*}{$\begin{array}{l}\text { SNP } \\
\mu \mathrm{M}\end{array}$} & \multirow[b]{3}{*}{ Sprays } & \multicolumn{4}{|c|}{$2017-2018$ Season } & \multicolumn{4}{|c|}{$2018-2019$ Season } \\
\hline & & \multicolumn{3}{|c|}{ Cultivars } & \multirow[b]{2}{*}{$\begin{array}{l}\text { SNP } \times \\
\text { Sprays }\end{array}$} & \multicolumn{3}{|c|}{ Cultivars } & \multirow[b]{2}{*}{$\begin{array}{l}\text { SNP } \times \\
\text { Sprays }\end{array}$} \\
\hline & & $\begin{array}{l}\text { Prukt } \\
\text { or F1 }\end{array}$ & Luna & $\begin{array}{c}\text { Rain } \\
\text { ball } \\
\text { F1 }\end{array}$ & & $\begin{array}{l}\text { Prukt } \\
\text { or F1 }\end{array}$ & Luna & $\begin{array}{c}\text { Rain } \\
\text { ball } \\
\text { F1 }\end{array}$ & \\
\hline \multirow[b]{2}{*}{0} & $\begin{array}{l}\text { Once } \\
\text { time }\end{array}$ & 1.92 & 1.76 & 1.62 & 1.77 & 1.79 & 1.60 & 1.53 & 1.64 \\
\hline & $\begin{array}{l}\text { Twice } \\
\text { time }\end{array}$ & 1.90 & 1.73 & 1.64 & 1.76 & 1.78 & 1.62 & 1.54 & 1.65 \\
\hline \multirow{2}{*}{50} & $\begin{array}{l}\text { Once } \\
\text { time }\end{array}$ & 2.42 & 2.31 & 1.99 & 2.24 & 2.19 & 1.87 & 1.78 & 1.95 \\
\hline & $\begin{array}{l}\text { Twice } \\
\text { time }\end{array}$ & 3.03 & 2.82 & 2.44 & 2.76 & 2.83 & 2.62 & 2.28 & 2.58 \\
\hline \multirow{2}{*}{100} & $\begin{array}{l}\text { Once } \\
\text { time }\end{array}$ & 2.88 & 2.61 & 2.38 & 2.62 & 2.68 & 2.42 & 2.29 & 2.46 \\
\hline & $\begin{array}{l}\text { Twice } \\
\text { time }\end{array}$ & 3.77 & 3.15 & 2.82 & 3.25 & 3.52 & 3.05 & 2.61 & 3.06 \\
\hline \multirow{2}{*}{150} & $\begin{array}{l}\text { Once } \\
\text { time }\end{array}$ & 3.61 & 2.90 & 2.64 & 3.05 & 3.30 & 2.85 & 2.46 & 2.87 \\
\hline & $\begin{array}{l}\text { Twice } \\
\text { time }\end{array}$ & 2.55 & 2.56 & 2.28 & 2.46 & 2.38 & 2.30 & 2.21 & 2.29 \\
\hline \multicolumn{2}{|c|}{ LSD 0.05} & & 0.09 & & 0.05 & & 0.22 & & 0.12 \\
\hline \multicolumn{2}{|c|}{ Effect of cultivars } & 2.76 & 2.48 & 2.23 & \multirow{2}{*}{$\begin{array}{l}\text { Effect } \\
\text { of SNP }\end{array}$} & 2.56 & 2.29 & 2.09 & \multirow{2}{*}{$\begin{array}{l}\text { Effect } \\
\text { of SNP }\end{array}$} \\
\hline LSI & 0.05 & & 0.04 & & & & 0.09 & & \\
\hline \multirow{4}{*}{$\begin{array}{l}\text { Cultiva } \\
\text { rs } \times \\
\text { SNP }\end{array}$} & 0 & 1.91 & 1.75 & 1.63 & 1.76 & 1.78 & 1.61 & 1.54 & 1.64 \\
\hline & 50 & 2.72 & 2.57 & 2.21 & 2.50 & 2.51 & 2.24 & 2.03 & 2.26 \\
\hline & 100 & 3.32 & 2.88 & 2.60 & 2.93 & 3.10 & 2.74 & 2.45 & 2.76 \\
\hline & 150 & 3.08 & 2.72 & 2.47 & 2.76 & 2.84 & 2.57 & 2.34 & 2.58 \\
\hline \multicolumn{2}{|c|}{ LSD 0.05} & & 0.07 & & 0.04 & & NS & & 0.09 \\
\hline & & & & & $\begin{array}{c}\text { Effect } \\
\text { of } \\
\text { Sprays }\end{array}$ & & $\mathrm{C}$ & & $\begin{array}{l}\text { Effect } \\
\text { of } \\
\text { Sprays }\end{array}$ \\
\hline \multirow{2}{*}{$\begin{array}{l}\text { Cultiva } \\
\text { rs } \times \\
\text { Sprays }\end{array}$} & $\begin{array}{l}\text { Once } \\
\text { time }\end{array}$ & 2.71 & 2.40 & 2.16 & 2.42 & 2.49 & 2.18 & 2.02 & 2.23 \\
\hline & $\begin{array}{l}\text { Twice } \\
\text { time }\end{array}$ & 2.81 & 2.57 & 2.30 & 2.56 & 2.63 & 2.40 & 2.16 & 2.40 \\
\hline \multicolumn{2}{|c|}{ LSD 0.05} & & NS & & 0.03 & & NS & & 0.06 \\
\hline
\end{tabular}


Al-Jarah et Basrah al. / J. Agric. Sci., 32 (2): 47-62, 2019

Table (6): Effect of cultivars, sprays and concentration of SNP on Na content (\%) in leaves.

\begin{tabular}{|c|c|c|c|c|c|c|c|c|c|}
\hline \multirow{3}{*}{$\begin{array}{l}\text { SNP } \\
\mu \mathrm{M}\end{array}$} & \multirow[b]{3}{*}{ Sprays } & \multicolumn{4}{|c|}{$2017-2018$ Season } & \multicolumn{4}{|c|}{$2018-2019$ Season } \\
\hline & & \multicolumn{3}{|c|}{ Cultivars } & \multirow[b]{2}{*}{$\begin{array}{l}\text { SNP } \times \\
\text { Sprays }\end{array}$} & \multicolumn{3}{|c|}{ Cultivars } & \multirow[b]{2}{*}{$\begin{array}{l}\text { SNP } \times \\
\text { Sprays }\end{array}$} \\
\hline & & $\begin{array}{l}\text { Prukt } \\
\text { or F1 }\end{array}$ & Luna & $\begin{array}{c}\text { Rain } \\
\text { ball } \\
\text { F1 }\end{array}$ & & $\begin{array}{l}\text { Prukt } \\
\text { or F1 }\end{array}$ & Luna & $\begin{array}{c}\text { Rain } \\
\text { ball } \\
\text { F1 }\end{array}$ & \\
\hline \multirow{2}{*}{0} & $\begin{array}{l}\text { Once } \\
\text { time }\end{array}$ & 1.693 & 1.835 & 2.035 & 1.854 & 1.879 & 1.990 & 2.113 & 1.994 \\
\hline & $\begin{array}{l}\text { Twice } \\
\text { time }\end{array}$ & 1.690 & 1.852 & 2.024 & 1.855 & 1.873 & 1.962 & 2.144 & 1.993 \\
\hline \multirow[b]{2}{*}{50} & $\begin{array}{l}\text { Once } \\
\text { time }\end{array}$ & 1.560 & 1.711 & 1.898 & 1.723 & 1.706 & 1.865 & 1.968 & 1.846 \\
\hline & $\begin{array}{l}\text { Twice } \\
\text { time }\end{array}$ & 1.207 & 1.424 & 1.631 & 1.421 & 1.339 & 1.634 & 1.816 & 1.597 \\
\hline \multirow{2}{*}{100} & $\begin{array}{l}\text { Once } \\
\text { time }\end{array}$ & 1.309 & 1.572 & 1.702 & 1.528 & 1.572 & 1.735 & 1.886 & 1.731 \\
\hline & $\begin{array}{l}\text { Twice } \\
\text { time }\end{array}$ & 0.986 & 1.280 & 1.439 & 1.235 & 1.215 & 1.396 & 1.509 & 1.373 \\
\hline \multirow{2}{*}{150} & $\begin{array}{l}\text { Once } \\
\text { time }\end{array}$ & 1.117 & 1.374 & 1.575 & 1.355 & 1.394 & 1.563 & 1.725 & 1.561 \\
\hline & $\begin{array}{l}\text { Twice } \\
\text { time }\end{array}$ & 0.871 & 1.079 & 1.310 & 1.087 & 1.088 & 1.201 & 1.409 & 1.233 \\
\hline \multicolumn{2}{|c|}{ LSD 0.05} & & $\mathrm{NS}$ & & 0.039 & & 0.052 & & 0.031 \\
\hline \multicolumn{2}{|c|}{ Effect of cultivars } & 1.304 & 1.516 & 1.702 & \multirow{2}{*}{$\begin{array}{c}\text { Effect } \\
\text { of SNP }\end{array}$} & 1.508 & 1.668 & 1.821 & \multirow{2}{*}{$\begin{array}{l}\text { Effect } \\
\text { of SNP }\end{array}$} \\
\hline $\mathrm{LS}$ & 0.05 & & 0.023 & & & & 0.024 & & \\
\hline \multirow{4}{*}{$\begin{array}{l}\text { Cultiva } \\
\text { rs } \times \\
\text { SNP }\end{array}$} & 0 & 1.691 & 1.843 & 2.029 & 1.855 & 1.876 & 1.976 & 2.128 & 1.993 \\
\hline & 50 & 1.383 & 1.568 & 1.765 & 1.572 & 1.523 & 1.749 & 1.892 & 1.721 \\
\hline & 100 & 1.148 & 1.426 & 1.570 & 1.381 & 1.393 & 1.566 & 1.697 & 1.552 \\
\hline & 150 & 0.994 & 1.227 & 1.442 & 1.221 & 1.241 & 1.382 & 1.567 & 1.397 \\
\hline \multicolumn{2}{|c|}{ LSD 0.05} & & 0.044 & & 0.027 & & 0.036 & & 0.021 \\
\hline & & & & & $\begin{array}{c}\text { Effect } \\
\text { of } \\
\text { Sprays }\end{array}$ & & & & $\begin{array}{l}\text { Effect } \\
\text { of } \\
\text { Sprays }\end{array}$ \\
\hline \multirow{2}{*}{$\begin{array}{l}\text { Cultiva } \\
\text { rs } \times \\
\text { Sprays }\end{array}$} & $\begin{array}{l}\text { Once } \\
\text { time }\end{array}$ & 1.420 & 1.623 & 1.802 & 1.615 & 1.638 & 1.788 & 1.923 & 1.783 \\
\hline & $\begin{array}{l}\text { Twice } \\
\text { time }\end{array}$ & 1.188 & 1.409 & 1.601 & 1.399 & 1.379 & 1.549 & 1.719 & 13549 \\
\hline \multicolumn{2}{|c|}{ LSD 0.05} & & NS & & 0.024 & & $\mathrm{NS}$ & & 0.020 \\
\hline
\end{tabular}


Table (7): Effect of cultivars, sprays and concentration of $\mathrm{SNP}$ on $\mathrm{K}^{+} / \mathrm{Na}^{+}$ratio in leaves.

\begin{tabular}{|c|c|c|c|c|c|c|c|c|c|}
\hline \multirow[b]{3}{*}{$\begin{array}{l}\text { SNP } \\
\mu \mathrm{M}\end{array}$} & \multirow[b]{3}{*}{ Sprays } & \multicolumn{4}{|c|}{$2017-2018$ Season } & \multicolumn{4}{|c|}{$2018-2019$ Season } \\
\hline & & \multicolumn{3}{|c|}{ Cultivars } & \multirow[b]{2}{*}{$\begin{array}{l}\text { SNP } \times \\
\text { Sprays }\end{array}$} & \multicolumn{3}{|c|}{ Cultivars } & \multirow[b]{2}{*}{$\begin{array}{l}\text { SNP } \times \\
\text { Sprays }\end{array}$} \\
\hline & & $\begin{array}{l}\text { Prukt } \\
\text { or F1 }\end{array}$ & Luna & $\begin{array}{c}\text { Rain } \\
\text { ball } \\
\text { F1 }\end{array}$ & & $\begin{array}{l}\text { Prukt } \\
\text { or F1 }\end{array}$ & Luna & $\begin{array}{c}\text { Rain } \\
\text { ball } \\
\text { F1 }\end{array}$ & \\
\hline \multirow{2}{*}{0} & $\begin{array}{l}\text { Once } \\
\text { time }\end{array}$ & 1.135 & 0.961 & 0.795 & 0.964 & 0.951 & 0.805 & 0.725 & 0.827 \\
\hline & $\begin{array}{l}\text { Twice } \\
\text { time }\end{array}$ & 1.123 & 0.937 & 0.808 & 0.956 & 0.952 & 0.826 & 0.719 & 0.832 \\
\hline \multirow{2}{*}{50} & $\begin{array}{l}\text { Once } \\
\text { time }\end{array}$ & 1.549 & 1.351 & 1.048 & 1.316 & 1.285 & 1.002 & 0.906 & 1.064 \\
\hline & $\begin{array}{l}\text { Twice } \\
\text { time }\end{array}$ & 2.512 & 1.983 & 1.497 & 1.997 & 2.112 & 1.603 & 1.254 & 1.656 \\
\hline \multirow{2}{*}{100} & $\begin{array}{l}\text { Once } \\
\text { time }\end{array}$ & 2.200 & 1.658 & 1.399 & 1.752 & 1.708 & 1.394 & 1.214 & 1.439 \\
\hline & $\begin{array}{l}\text { Twice } \\
\text { time }\end{array}$ & 3.832 & 2.464 & 1.962 & 2.753 & 2.900 & 2.189 & 1.728 & 2.272 \\
\hline \multirow{2}{*}{150} & $\begin{array}{l}\text { Once } \\
\text { time }\end{array}$ & 3.233 & 2.111 & 1.679 & 2.341 & 2.369 & 1.820 & 1.427 & 1.872 \\
\hline & $\begin{array}{l}\text { Twice } \\
\text { time }\end{array}$ & 2.931 & 2.372 & 1.744 & 2.349 & 2.184 & 1.912 & 1.569 & 1.888 \\
\hline \multicolumn{2}{|c|}{ LSD 0.05} & & 0.150 & & 0.090 & & 0.129 & & 0.076 \\
\hline \multicolumn{2}{|c|}{ Effect of cultivars } & 2.314 & 1.729 & 1.366 & Effect & 1.808 & 1.444 & 1.193 & Effect \\
\hline \multicolumn{2}{|c|}{ LSD 0.05} & & 0.071 & & of SNP & & 0.053 & & of SNP \\
\hline \multirow{4}{*}{$\begin{array}{c}\text { Cultiva } \\
\text { rs } \times \\
\text { SNP }\end{array}$} & 0 & 1.129 & 0.949 & 0.802 & 0.960 & 0.952 & 0.815 & 0.722 & 0.829 \\
\hline & 50 & 2.030 & 1.667 & 1.273 & 1.656 & 1.698 & 1.302 & 1.080 & 1.360 \\
\hline & 100 & 3.016 & 2.061 & 1.681 & 2.253 & 2.304 & 1.791 & 1.471 & 1.855 \\
\hline & 150 & 3.082 & 2.242 & 1.711 & 2.345 & 2.277 & 1.866 & 1.498 & 1.880 \\
\hline \multicolumn{2}{|c|}{ LSD 0.05} & & 0.104 & & 0.060 & & 0.091 & & 0.055 \\
\hline & & & & & $\begin{array}{c}\text { Effect } \\
\text { of } \\
\text { Sprays }\end{array}$ & & & & $\begin{array}{c}\text { Effect } \\
\text { of } \\
\text { sprays }\end{array}$ \\
\hline \multirow{2}{*}{$\begin{array}{l}\text { Cultiva } \\
\text { rs } \times \\
\text { Sprays }\end{array}$} & $\begin{array}{l}\text { Once } \\
\text { time }\end{array}$ & 2.029 & 1.520 & 1.230 & 1.593 & 1.578 & 1.255 & 1.068 & 1.300 \\
\hline & $\begin{array}{l}\text { Twice } \\
\text { time }\end{array}$ & 2.600 & 1.939 & 1.503 & 2.014 & 2.037 & 1.632 & 1.318 & 1.662 \\
\hline \multicolumn{2}{|c|}{ LSD 0.05} & & 0.090 & & 0.062 & & 0.064 & & 0.042 \\
\hline
\end{tabular}


Table (8): Effect of cultivars, sprays and concentration of SNP on Cl content (\%) in leaves.

\begin{tabular}{|c|c|c|c|c|c|c|c|c|c|}
\hline \multirow{3}{*}{$\begin{array}{l}\text { SNP } \\
\mu \mathrm{M}\end{array}$} & \multirow[b]{3}{*}{ Sprays } & \multicolumn{4}{|c|}{$2017-2018$ Season } & \multicolumn{4}{|c|}{$2018-2019$ Season } \\
\hline & & \multicolumn{3}{|c|}{ Cultivars } & \multirow[b]{2}{*}{$\begin{array}{l}\text { SNP } \times \\
\text { Sprays }\end{array}$} & \multicolumn{3}{|c|}{ Cultivars } & \multirow[b]{2}{*}{$\begin{array}{l}\text { SNP } \times \\
\text { Sprays }\end{array}$} \\
\hline & & $\begin{array}{l}\text { Prukt } \\
\text { or F1 }\end{array}$ & Luna & $\begin{array}{c}\text { Rain } \\
\text { ball } \\
\text { F1 }\end{array}$ & & $\begin{array}{l}\text { Prukt } \\
\text { or F1 }\end{array}$ & Luna & $\begin{array}{c}\text { Rain } \\
\text { ball } \\
\text { F1 }\end{array}$ & \\
\hline \multirow{2}{*}{0} & $\begin{array}{l}\text { Once } \\
\text { time }\end{array}$ & 4.53 & 4.69 & 4.79 & 4.67 & 4.83 & 4.97 & 5.08 & 4.96 \\
\hline & $\begin{array}{l}\text { Twice } \\
\text { time }\end{array}$ & 4.42 & 4.56 & 4.50 & 4.49 & 4.69 & 4.83 & 4.77 & 4.76 \\
\hline \multirow{2}{*}{50} & $\begin{array}{l}\text { Once } \\
\text { time }\end{array}$ & 4.07 & 4.24 & 4.27 & 4.20 & 4.33 & 4.49 & 4.54 & 4.45 \\
\hline & $\begin{array}{l}\text { Twice } \\
\text { time }\end{array}$ & 3.53 & 3.93 & 3.77 & 3.75 & 3.75 & 4.16 & 3.99 & 3.97 \\
\hline \multirow{2}{*}{100} & $\begin{array}{l}\text { Once } \\
\text { time }\end{array}$ & 3.60 & 3.78 & 3.72 & 3.70 & 3.83 & 3.99 & 3095 & 3.92 \\
\hline & $\begin{array}{l}\text { Twice } \\
\text { time }\end{array}$ & 2.83 & 3.30 & 3.26 & 3.13 & 2.99 & 3.48 & 3.45 & 3.31 \\
\hline \multirow{2}{*}{150} & $\begin{array}{l}\text { Once } \\
\text { time }\end{array}$ & 3.23 & 3.43 & 3.49 & 3.38 & 3.43 & 3.62 & 3.67 & 3.57 \\
\hline & $\begin{array}{l}\text { Twice } \\
\text { time }\end{array}$ & 2.89 & 3.32 & 3.21 & 3.14 & 3.04 & 3.52 & 3.39 & 3.32 \\
\hline \multicolumn{2}{|c|}{ LSD 0.05} & & 0.13 & & 0.08 & & 0.13 & & 0.08 \\
\hline \multicolumn{2}{|c|}{ Effect of cultivars } & 3.64 & 3.91 & 3.88 & \multirow{2}{*}{$\begin{array}{l}\text { Effect } \\
\text { of SNP }\end{array}$} & 3.86 & 4.13 & 4.10 & \multirow{2}{*}{$\begin{array}{l}\text { Effect } \\
\text { of SNP }\end{array}$} \\
\hline LSI & 0.05 & & 0.06 & & & & 0.07 & & \\
\hline \multirow{4}{*}{$\begin{array}{l}\text { Cultiva } \\
\text { rs } \times \\
\text { SNP }\end{array}$} & 0 & 4.47 & 4.63 & 4.65 & 4.58 & 4.76 & 4.90 & 4.93 & 4.86 \\
\hline & 50 & 3.80 & 4.09 & 4.02 & 3.97 & 4.04 & 4.33 & 4.26 & 4.21 \\
\hline & 100 & 3.21 & 3.54 & 3.49 & 3.41 & 3.41 & 3.73 & 3.70 & 3.61 \\
\hline & 150 & 3.06 & 3.38 & 3.35 & 3.26 & 3.23 & 3.57 & 3.53 & 3.45 \\
\hline \multicolumn{2}{|c|}{ LSD 0.05} & & NS & & 0.05 & & 0.10 & & 0.05 \\
\hline & & & & & $\begin{array}{c}\text { Effect } \\
\text { of } \\
\text { Sprays }\end{array}$ & & & & $\begin{array}{l}\text { Effect } \\
\text { of } \\
\text { sprays } \\
\end{array}$ \\
\hline \multirow{2}{*}{$\begin{array}{l}\text { Cultiva } \\
\text { rs } \times \\
\text { Sprays }\end{array}$} & $\begin{array}{l}\text { Once } \\
\text { time }\end{array}$ & 3.86 & 4.04 & 4.07 & 3.99 & 4.10 & 4.27 & 4.31 & 4.23 \\
\hline & $\begin{array}{l}\text { Twice } \\
\text { time }\end{array}$ & 3.42 & 3.78 & 3.69 & 3.63 & 3.62 & 4.00 & 3.90 & 3.84 \\
\hline \multicolumn{2}{|c|}{ LSD 0.05} & & NS & & 0.06 & & $\mathrm{NS}$ & & 0.06 \\
\hline
\end{tabular}


Al-Jarah et Basrah al. / J. Agric. Sci., 32 (2): 47-62, 2019

Table (9): Effect of cultivars, sprays and concentration of SNP on S content (\%) in leaves.

\begin{tabular}{|c|c|c|c|c|c|c|c|c|c|}
\hline \multirow{3}{*}{$\begin{array}{l}\text { SNP } \\
\mu \mathrm{M}\end{array}$} & \multirow[b]{3}{*}{ Sprays } & \multicolumn{4}{|c|}{$2017-2018$ Season } & \multicolumn{4}{|c|}{$2018-2019$ Season } \\
\hline & & \multicolumn{3}{|c|}{ Cultivars } & \multirow[b]{2}{*}{$\begin{array}{l}\text { SNP } \times \\
\text { Sprays }\end{array}$} & \multicolumn{3}{|c|}{ Cultivars } & \multirow[b]{2}{*}{$\begin{array}{l}\text { SNP } \times \\
\text { Sprays }\end{array}$} \\
\hline & & $\begin{array}{l}\text { Prukt } \\
\text { or F1 }\end{array}$ & Luna & $\begin{array}{c}\text { Rain } \\
\text { ball } \\
\text { F1 }\end{array}$ & & $\begin{array}{l}\text { Prukt } \\
\text { or F1 }\end{array}$ & Luna & $\begin{array}{c}\text { Rain } \\
\text { ball } \\
\text { F1 }\end{array}$ & \\
\hline \multirow[b]{2}{*}{0} & $\begin{array}{l}\text { Once } \\
\text { time }\end{array}$ & 0.417 & 0.390 & 0.375 & 0.394 & 0.404 & 0.377 & 0.367 & 0.383 \\
\hline & $\begin{array}{l}\text { Twice } \\
\text { time }\end{array}$ & 0.397 & 0.391 & 0.370 & 0.386 & 0.387 & 0.382 & 0.360 & 0.376 \\
\hline \multirow{2}{*}{50} & $\begin{array}{l}\text { Once } \\
\text { time }\end{array}$ & 0.497 & 0.460 & 0.450 & 0.469 & 0.486 & 0.448 & 0.429 & 0.454 \\
\hline & $\begin{array}{l}\text { Twice } \\
\text { time }\end{array}$ & 0.561 & 0.551 & 0.541 & 0.551 & 0.542 & 0.540 & 0.529 & 0.537 \\
\hline \multirow{2}{*}{100} & $\begin{array}{l}\text { Once } \\
\text { time }\end{array}$ & 0.551 & 0.542 & 0.525 & 0.539 & 0.533 & 0.530 & 0.511 & 0.525 \\
\hline & $\begin{array}{l}\text { Twice } \\
\text { time }\end{array}$ & 0.731 & 0.681 & 0.620 & 0.677 & 0.717 & 0.670 & 0.604 & 0.664 \\
\hline \multirow{2}{*}{150} & $\begin{array}{l}\text { Once } \\
\text { time }\end{array}$ & 0.626 & 0.639 & 0.582 & 0.617 & 0.611 & 0.632 & 0.566 & 0.600 \\
\hline & $\begin{array}{l}\text { Twice } \\
\text { time }\end{array}$ & 0.675 & 0.648 & 0.601 & 0.641 & 0.659 & 0.639 & 0.586 & 0.628 \\
\hline \multicolumn{2}{|c|}{ LSD 0.05} & & 0.028 & & 0.016 & & 0.020 & & 0.012 \\
\hline \multicolumn{2}{|c|}{ Effect of cultivars } & 0.557 & 0.538 & 0.508 & Effect & 0.542 & 0.526 & 0.494 & Effect \\
\hline \multicolumn{2}{|c|}{ LSD 0.05} & & 0.013 & & of SNP & & 0.008 & & of SNP \\
\hline \multirow{4}{*}{$\begin{array}{l}\text { Cultiva } \\
\text { rs } \times \\
\text { SNP }\end{array}$} & 0 & 0.407 & 0.390 & 0.373 & 0.390 & 0.396 & 0.379 & 0.364 & 0.380 \\
\hline & 50 & 0.529 & 0.506 & 0.495 & 0.510 & 0.514 & 0.494 & 0.479 & 0.496 \\
\hline & 100 & 0.641 & 0.612 & 0.573 & 0.608 & 0.625 & 0.600 & 0.558 & 0.594 \\
\hline & 150 & 0.651 & 0.643 & 0.592 & 0.629 & 0.635 & 0.631 & 0.576 & 0.614 \\
\hline \multicolumn{2}{|c|}{ LSD 0.05} & & 0.020 & & 0.012 & & 0.014 & & 0.009 \\
\hline & & & & & $\begin{array}{c}\text { Effect } \\
\text { of } \\
\text { Sprays }\end{array}$ & & & & $\begin{array}{l}\text { Effect } \\
\text { of } \\
\text { Sprays }\end{array}$ \\
\hline \multirow{2}{*}{$\begin{array}{l}\text { Cultiva } \\
\text { rs } \times \\
\text { Sprays }\end{array}$} & $\begin{array}{l}\text { Once } \\
\text { time }\end{array}$ & 0.523 & 0.508 & 0.482 & 0.504 & 0.509 & 0.495 & 0.468 & 0.491 \\
\hline & $\begin{array}{l}\text { Twice } \\
\text { time }\end{array}$ & 0.591 & 0.568 & 0.533 & 0.564 & 0.576 & 0.558 & 0.520 & 0.551 \\
\hline \multicolumn{2}{|c|}{ LSD 0.05} & & NS & & 0.008 & & NS & & 0.007 \\
\hline
\end{tabular}


Table (10): Effect of cultivars, sprays and concentration of SNP on Fe content (mg. $\mathrm{Kg}^{-1}$ D W) in leaves.

\begin{tabular}{|c|c|c|c|c|c|c|c|c|c|}
\hline \multirow{3}{*}{$\begin{array}{l}\text { SNP } \\
\mu \mathrm{M}\end{array}$} & \multirow[b]{3}{*}{ Sprays } & \multicolumn{4}{|c|}{$2017-2018$ Season } & \multicolumn{4}{|c|}{2018 - 2019 Season } \\
\hline & & \multicolumn{3}{|c|}{ Cultivars } & \multirow[b]{2}{*}{$\begin{array}{l}\text { SNP } \times \\
\text { Sprays }\end{array}$} & \multicolumn{3}{|c|}{ Cultivars } & \multirow[b]{2}{*}{$\begin{array}{l}\text { SNP } \times \\
\text { Sprays }\end{array}$} \\
\hline & & $\begin{array}{c}\text { Pruktor } \\
\text { F1 }\end{array}$ & Luna & $\begin{array}{c}\text { Rain } \\
\text { ball } \\
\text { F1 }\end{array}$ & & $\begin{array}{l}\text { Prukt } \\
\text { or F1 }\end{array}$ & Luna & $\begin{array}{c}\text { Rain } \\
\text { ball } \\
\text { F1 } \\
\end{array}$ & \\
\hline \multirow{2}{*}{0} & $\begin{array}{l}\text { Once } \\
\text { time }\end{array}$ & 45.32 & 40.78 & 45.32 & 40.93 & 42.77 & 38.00 & 34.71 & 38.49 \\
\hline & $\begin{array}{l}\text { Twice } \\
\text { time }\end{array}$ & 44.46 & 40.71 & 44.46 & 40.81 & 41.37 & 38.90 & 35.00 & 38.42 \\
\hline \multirow{2}{*}{50} & $\begin{array}{l}\text { Once } \\
\text { time }\end{array}$ & 51.36 & 45.58 & 51.36 & 46.57 & 47.97 & 42.77 & 40.21 & 43.65 \\
\hline & $\begin{array}{l}\text { Twice } \\
\text { time }\end{array}$ & 66.18 & 59.59 & 66.18 & 60.61 & 62.07 & 51.23 & 51.97 & 55.09 \\
\hline \multirow{2}{*}{100} & $\begin{array}{l}\text { Once } \\
\text { time }\end{array}$ & 60.15 & 57.44 & 60.15 & 57.26 & 55.60 & 48.02 & 47.77 & 50.47 \\
\hline & $\begin{array}{l}\text { Twice } \\
\text { time }\end{array}$ & 77.03 & 69.60 & 77.03 & 69.95 & 74.36 & 61.06 & 59.45 & 64.96 \\
\hline \multirow{2}{*}{150} & $\begin{array}{l}\text { Once } \\
\text { time }\end{array}$ & 69.32 & 64.72 & 69.32 & 64.59 & 65.11 & 53.04 & 53.93 & 57.36 \\
\hline & $\begin{array}{l}\text { Twice } \\
\text { time }\end{array}$ & 67.31 & 60.36 & 67.31 & 61.53 & 62.87 & 54.00 & 54.49 & 57.12 \\
\hline \multicolumn{2}{|c|}{ LSD 0.05} & & 1.91 & & 1.14 & & 1.44 & & 0.97 \\
\hline \multicolumn{2}{|c|}{ Effect of cultivars } & 60.14 & 54.85 & 50.86 & \multirow{2}{*}{$\begin{array}{c}\text { Effect } \\
\text { of SNP }\end{array}$} & 56.52 & 48.38 & 47.19 & \multirow{2}{*}{$\begin{array}{c}\text { Effect } \\
\text { of SNP }\end{array}$} \\
\hline LSI & 0.05 & & 0.64 & & & & 0.58 & & \\
\hline \multirow{4}{*}{$\begin{array}{c}\text { Cultiva } \\
\text { rs } \times \\
\text { SNP }\end{array}$} & 0 & 44.89 & 40.74 & 36.99 & 40.87 & 42.07 & 38.45 & 34.85 & 38.46 \\
\hline & 50 & 58.77 & 52.58 & 49.43 & 53.59 & 55.02 & 47.00 & 46.09 & 49.37 \\
\hline & 100 & 68.59 & 63.52 & 58.69 & 63.60 & 64.98 & 54.54 & 53.61 & 57.71 \\
\hline & 150 & 68.31 & 62.54 & 58.34 & 63.06 & 63.99 & 53.52 & 54.21 & 57.24 \\
\hline \multicolumn{2}{|c|}{ LSD 0.05} & & NS & & 0.82 & & 0.86 & & 0.50 \\
\hline & & & & & $\begin{array}{c}\text { Effect } \\
\text { of } \\
\text { Sprays }\end{array}$ & & & & $\begin{array}{c}\text { Effect } \\
\text { of } \\
\text { Sprays }\end{array}$ \\
\hline \multirow{2}{*}{$\begin{array}{c}\text { Cultiva } \\
\text { rs } \times \\
\text { Sprays }\end{array}$} & $\begin{array}{l}\text { Once } \\
\text { time }\end{array}$ & 56.53 & 52.13 & 48.36 & 52.34 & 52.86 & 45.46 & 44.16 & 47.49 \\
\hline & $\begin{array}{l}\text { Twice } \\
\text { time }\end{array}$ & 63.74 & 57.56 & 53.37 & 58.23 & 60.17 & 51.30 & 50.23 & 53.90 \\
\hline \multicolumn{2}{|c|}{ LSD 0.05} & & 0.89 & & 0.65 & & NS & & 0.84 \\
\hline
\end{tabular}

\section{Conclusions}

The results showed that sodium nitroprusside (SNP) at $100 \mu \mathrm{M}$ Sprays twice-time enhanced the tolerance of cabbage cv. Pruktor F1 to salt stress by improving accumulation of $\mathrm{P}, \mathrm{K}, \mathrm{Fe}$ and $\mathrm{S}$ and increase $\mathrm{K}+/ \mathrm{Na}+$ ratio in leaves.

\section{Acknowledgements}

Authors would like to thank Mr. Jameel H.

Hiji from Department of Horticulture and 
Landscape Design for his assistance in proofreading the manuscript.

Conflict of interest: The authors declare that they have no conflict of interest.

\section{References}

Agricultural Statistics Directorate (2018). Central Statistical Organization. Min. Planning, Iraq.

Al-Taey, D.K.A.; Al-Janabi, A.H. \& Rachid, A.M. (2017). Effect of water salinity and organic \& mineral fertilizers on the growth and some contents of leave nutrients of cabbage (Brassica oleracea var. capitate L.). J. Univ. Babylon Pure Appl. Sci., 25(6): 2046- 2064.

Arasimowicz, M. \& Floryszak-Wieczorek, J. (2007). Nitric oxide as a bioactive signaling molecule in plant stress responses. Plant Sci., 172: 876-887.

Ashley, M.K.; Grant, M. \& Grabov, A. (2006). Plant responses to potassium deficiencies: a role for potassium transport proteins. J. Exp. Bot., 57(2): 425-436.

Ayers, R.S. \& Wescot, D.V. (1985). Water quality for agriculture. FAO Irrigation and Drainage Paper 29. FAO, Roma, 174pp.

Belgini, M.V. \& Lamattina, L. (1999). Nitric oxide counteracts cytotoxic process mediated by reactive oxygen species in plant tissues. Planta, 208: 337-44.

Black, C.A. (1965). Method of Soil Analysis. Part (1). Physical properties.
Am. Soc. Agron. Inc. Publisher, Madison, Wisconsin: 770pp..

Connolly, E.; Fett, J.P. \& Guerinot, M. L. (2002). Expression of the IRT1 metal transporter is controlled by metals at the levels of transcript and protein accumulation. Plant Cell, 14: 13471357.

Cuartero, J. \& Fernande-Munoz, R. (1999). Tomato and salinity. Sci. Horticult., 78: 83-125.

Dean-Drummond, C.E. (1986). A comparison of regulatory effects of chloride on nitrate uptake, and on chloride uptake into Pisum sativum seedlings. Physiol. Plant, 66: 115-126.

Duogrameji, J. \& Al-Rawi, J. (1972). Partical size dispersion method in some Iraqi soil. Zeitschrift für Pflanzenährung und Bodenkunde, 131(1): 38-42.

Furman, N.H. (1962). Standard Method of Chemical Analysis. $6^{\text {th }}$ ed. D. Van Nostrand, Co., Inc., Princeton, N.J.: 365pp.

Graziano, M.; Beligni, M.V.L. \& Lamattina, L. (2002). Nitric oxide improves internal iron availability in plants. Plant Physiol., 130: 1852-1859.

Hayat, S.; Yadav, S.; Alyemeni, M.N. \& Ahmad, A. (2014). Effect of sodium nitroprusside on the germination and antioxidant activities of tomato (Lycopersicon esculentum Mill). Bulg. J. Agric. Sci., 20(1): 156-160.

Haynes, R.J. (1980). Ion exchange properties of roots and ionic interactions within the root apoplasm: Their role in 
ion accumulation by plants. Bot. Rev., 46(1): 75-99.

Jabeen, N. \& Ahmad, R. (2011). Foliar application of potassium nitrate affects the growth and nitrate reductase activity in sunflower and safflower leaves under salinity. Not. Bot. Hort. Agrob., 39 (2): 172-178.

Jackson, M.L. (1958). Soil chemical analysis. Prentice Hall. Inc. Englewood Cliffs, N. J.: 598pp.

Lin, H.; Sandra, S.S. \& Schumaker, K.S. (1997). Salt sensitivity and the activities of the H-ATPase in cotton seedlings. Crop Sci., 37: 190-197.

Lopez-Cantarero, I.; Ruiz, J.M.; Hernandez, J. \& Romero, L. (1997). Nitrogen metabolism and yield response to increase in nitrogen-phosphorus fertilization; Improvement in greenhouse cultivation of eggplant (Solanum melongena). J. Agric. Food Chem., 45: 4227-4231.

Martinez, V. \& Lauchli, A. (1994) . Saltinduced inhibition of phosphate uptake in plants of cotton (Gossypium hirsutum L.). New Phytol., 125: 609-614.

Meloni, D.A.; Gulotta, M.R.; Martinez, C. A. \& Oliva, M.A. ( 2004). The effects of salt stress on growth, nitrate reduction and proline and glycinebetaine accumulation in Prosopis alba. Braz. J. Plant Physiol., 16(1): 39-46.

Molassiotis, A.; Tanou, G. \& Diamantidis, G. (2010). NO says more than 'YES' to salt tolerance: salt priming and systemic nitric oxide signaling in plants. Plant Signalling and Behavior, 5: 209-212.

Munns, R. \& Tester, M. (2008). Mechanisms of salinity tolerance. Ann. Rev. Plant Biol., 59: 651-681.

Nabi, R.B.S.; Tayade, R.; Hussain, A.; Kulkarni, K.; Imran, Q.M.; Mun, B.G. \& Yun, B.W. (2019). Nitric oxide regulates plant responses to drought, salinity, and heavy metal stress. Environ. Exp. Bot., 161: 120-133.

Novozamsky, I. \& Eck, R. (1977). Total sulphur determination in plant material. Fresen. J. Anal. Chem., 286 (5): 367368.

Page, A.L.; Miller, R.H. \& Keeney, D.R. (1982). Method of soil and analysis Part 2, $2^{\text {nd }}$ ed, Agron. 9. Publisher, Madison, Wisconsin: 1158pp.

Pessarakli, M. (1999). Handbook of Plant of Crop Stress. $2^{\text {nd }}$ en., Univ. CRC Press, Boca Raton: 1254pp.

Phocaides, A. (2001). Handbook on perssurized irrigation techniques FAO consultant, Rome, chapter 7, Water quality for irrigation.

Reddy, M.P. \& Vora, A.B. (1985). Effect of salinity on protein metabolism in bajra (Pennisetum typhoides $\mathrm{S}$ and $\mathrm{H}$ ) leaves. Indian J. Plant Physiol., 28: 190195.

Richards, A. (1954). Diagnosis and improvement of saline and alkali soils. Agriculture Handbook, No. 60, USDA, Washington: 160pp.

Santisree, P.; Adimulam, S. S.; Sharma, K.; Bhatnagar-Mathur, P. \& Sharma, K. 
K. (2019). Insights Into the Nitric Oxide Mediated Stress tolerance in plant. 385406. In Khan, I.M.R.; Reddy, P.S. Ferrante, A. \& Khan, N. (Eds.). Plant Signaling Molecular: Role and Regulation Under Stressful Environments. Elsevier: 596pp.

WHO, World Health Organization. (2015). WHO Model List of Essential Medicines (April 2015). $19^{\text {th }}$ list. www.who.int/selection_medicines/com mittees/expert/20/EML_2015_Final_am ended_AUG2015.pdf?au=1.
Zhao, L.Q.; Zhang, F. ; Guo, J.K.; Yang, Y. L.; Li, B.B. \& Zhang, L. X. (2004). Nitric oxide functions as a signal in salt resistance in the calluses from two ecotypes of reed. Plant Physiol., 134: 849-857.

Zhu, J.K. (2003). Regulation of ion homeostasis under salt stress. Curr. Opin. Plant Biol., 6: 441-445. 